\title{
Optical coherence tomography of pneumatic displacement of optic disc pit maculopathy
}

\author{
Harvey Lincoff, Ingrid Kreissig
}

\begin{abstract}
Background-The authors have previously concluded that fluid from an optic disc pit creates an inner layer separation (ILS) of the retina. An outer layer detachment (OLD) centred on the macula is a secondary phenomenon that causes a dense central scotoma. Pneumatic displacement of the OLD effects an improvement in central vision. Pathology to confirm these conclusions is lacking. Intraretinal images obtained by optical coherence tomography (OCT), however, are confirmatory.

Methods-Three patients with optic disc pit maculopathy were studied with stereoscopic photographs, visual fields, and OCT before and after intravitreal gas was injected to displace the central retinal elevation to below the inferior temporal vascular arcade.

Results-Preoperatively, OCT demonstrated an ILS that connected with the optic disc pit. External to it was an OLD that centred on the fovea and did not connect with the optic disc pit. Pneumatic displacement of the OLD was accompanied by an improvement in central vision. Long term follow up indicates that the effect of displacement may be temporary. Conclusion-OCT confirmed the two layer structure of optic disc pit maculopathy and that the improvement in central vision after pneumatic displacement coincides with a reattachment of the OLD in the macula. It also supports the hypothesis that the ILS, which persists, provides a conduit for the continuous flow of fluid from the pit to the displaced retinal elevation.
\end{abstract}

(Br f Ophthalmol 1998;82:367-372)

Department of Ophthalmology, New York Hospital-Cornell Medical Center, New York, USA

H Lincoff

Department of Ophthalmology III, University of Tübingen, Germany I Kreissig

Correspondence to: Professor Ingrid Kreissig, Univ-Augenklinik,

Schleichstrasse 12, D-72076

Tübingen, Germany.

Accepted for publication 6 November 1997
In 1988 it was proposed that the fluid emanating from an optic disc pit was creating a schisis-like separation of the inner layers (ILS) of the retina. A detachment of the outer layers (OLD) from pigment epithelium was a secondary process that began in the centre of the macula and did not connect with the optic disc pit. ${ }^{1}$ In a subsequent report we described that improvement in central vision could be obtained by pneumatic displacement of the OLD. ${ }^{2}$ Pathological confirmation of the two layer structure and of displacement by an intraocular gas bubble is lacking.

Optical coherence tomography (OCT) is a new technique for imaging the retina that utilises optical reflectivity to obtain cross sectional retinal images of the retina. ${ }^{34} \mathrm{We}$ report the course of three patients with an optic disc pit and clinical and stereoscopic evidence of optic disc pit maculopathy for whom OCT was performed before and after an intravitreal gas injection. The gas displaced the OLD from the macula in two of them.

\section{Materials and methods}

The fundus of the three patients with optic disc pit maculopathy was examined preoperatively with indirect ophthalmoscopy and slit lamp biomicroscopy. Corrected visual acuity was recorded and the central visual field was plotted on a 1 metre grey tangent screen. Colour and red-free stereophotographs were taken of the posterior pole. OCT of the posterior pole was made in a horizontal plane through the macula and the disc pit and vertically through the macula.

After operation the patients were examined, photographed, and had visual fields tested at weekly intervals for 1 month, at 3 month intervals for 1 year, and yearly thereafter. OCT was performed 1 week and 4 weeks postoperatively except for patient no 3 who had his only OCT done 5 years after the gas displacement procedure.

The gas injection was performed under retrobulbar anaesthesia. Before injection decompression of the eye was obtained by inserting a parabulbar balloon subconjunctivally to the equator in the inferonasal quadrant and expanding it until the central retinal artery pulsated. $^{56}$ After 1 hour the balloon was deflated and $0.4-0.6 \mathrm{ml}$ of undiluted perfluoroethane $\left(\mathrm{C}_{2} \mathrm{~F}_{6}\right)$, a gas that expands 3.3 times, was injected into the vitreous through the pars plana. ${ }^{7}$ The central retinal artery was examined for patency.

After the gas injection the patients were asked to maintain a position that enabled them to gaze between their heels while sitting or standing. At 30 minute intervals they were instructed to slowly rotate their gaze from straight ahead to between their heels. The rotations were intended to massage the fluid beneath the retina from the posterior pole to below the inferior vascular arcade. For sleeping they were asked to lie prone with their head off the edge of two pillows and their nose pointed approximately $30^{\circ}$ off the vertical. The positions were rehearsed with the patients. The rotations were continued for 2-3 weeks which was as long as the bubble was large enough to be therapeutically useful.

\section{Case reports}

PATIENT 1

A 53 year old man presented with a 1 week history of loss of vision in his right eye. The 

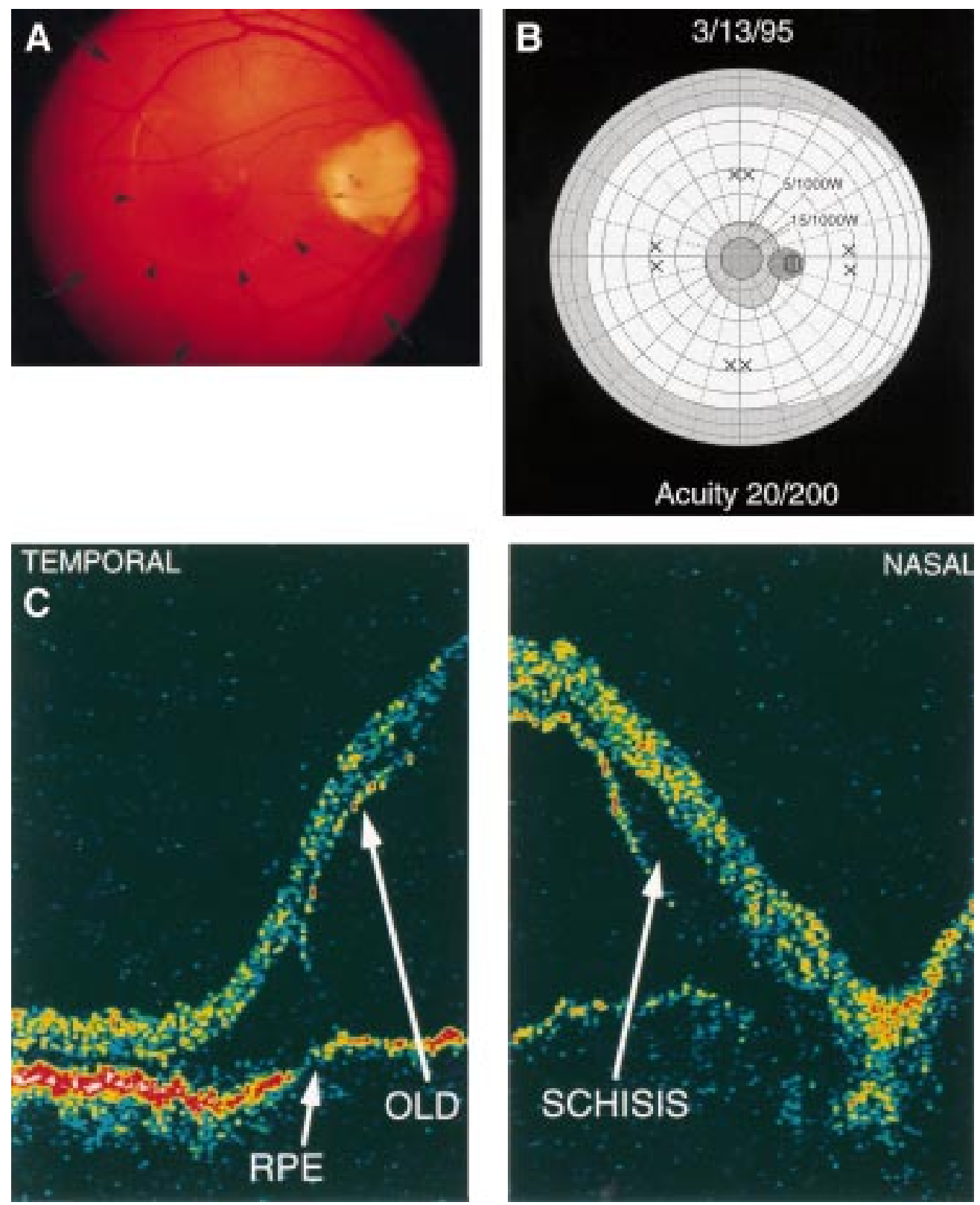

Figure 1 (A) The posterior pole of an eye with an optic disc pit maculopathy of 1 week's duration (patient no 1): a transparent ILS connects with the pit and extends to the superior and inferior vascular arcades (short arrows; outer circle). A relatively dense OLD (arrowheads) centres on the macula. (B) Central visual field of the patient whose eye is depicted in (A). There is a dense central scotoma $(15 / 1000 \mathrm{~W})$ which is consistent with the OLD. It is surrounded by a less dense scotoma $(5 / 1000 \mathrm{~W})$ which is consistent with the extent of the ILS. W= white. (C) OCT of the posterior pole of the eye depicted in (A). A schisis-like cavity is evident between the OLD and the internal layer separation. RPE = retinal pigment epithelium; OLD = outer layer detachment; - horizontal section through macula.

corrected visual acuity was $20 / 200$. The right eye had an inferotemporal optic nerve pit. Extending from the pit, there was an ILS that on biomicroscopy appeared relatively transparent. It extended to the upper and lower temporal vascular arcades and about 1.5 disc diameters temporal to the fovea. External to it was an opaque OLD of lesser extent, which centred on the macula. A visual field demonstrated a central scotoma to a $5 / 1000$ white test object that subtended $20^{\circ}$ of the central field and was consistent with the extent of the ILS. Within it was a denser scotoma, $15^{\circ}$ in diameter, that was consistent with the OLD. The OCT demonstrated an ILS and an OLD with a schisis-like space between them (Fig 1).
For pneumatic displacement of the OLD, $0.6 \mathrm{ml}$ of undiluted perfluoroethane $\left(\mathrm{C}_{2} \mathrm{~F}_{6}\right)$ was injected into the vitreous. The gas bubble expanded to $2 \mathrm{ml}$ in 3 days and the meniscus was then at the superior margin of the disc. The patient was instructed about position and rotation exercises. At 1 week the meniscus of the gas bubble was still at the top of the disc. The central retina was less elevated because much of the intraretinal and subretinal fluid had been displaced inferiorly. The ILS extended inferior to the inferior vascular arcade and some of the OLD was below the level of the inferior margin of the optic disc. The visual field was consistent with the displacements. The visual acuity had improved to 20/100. The 

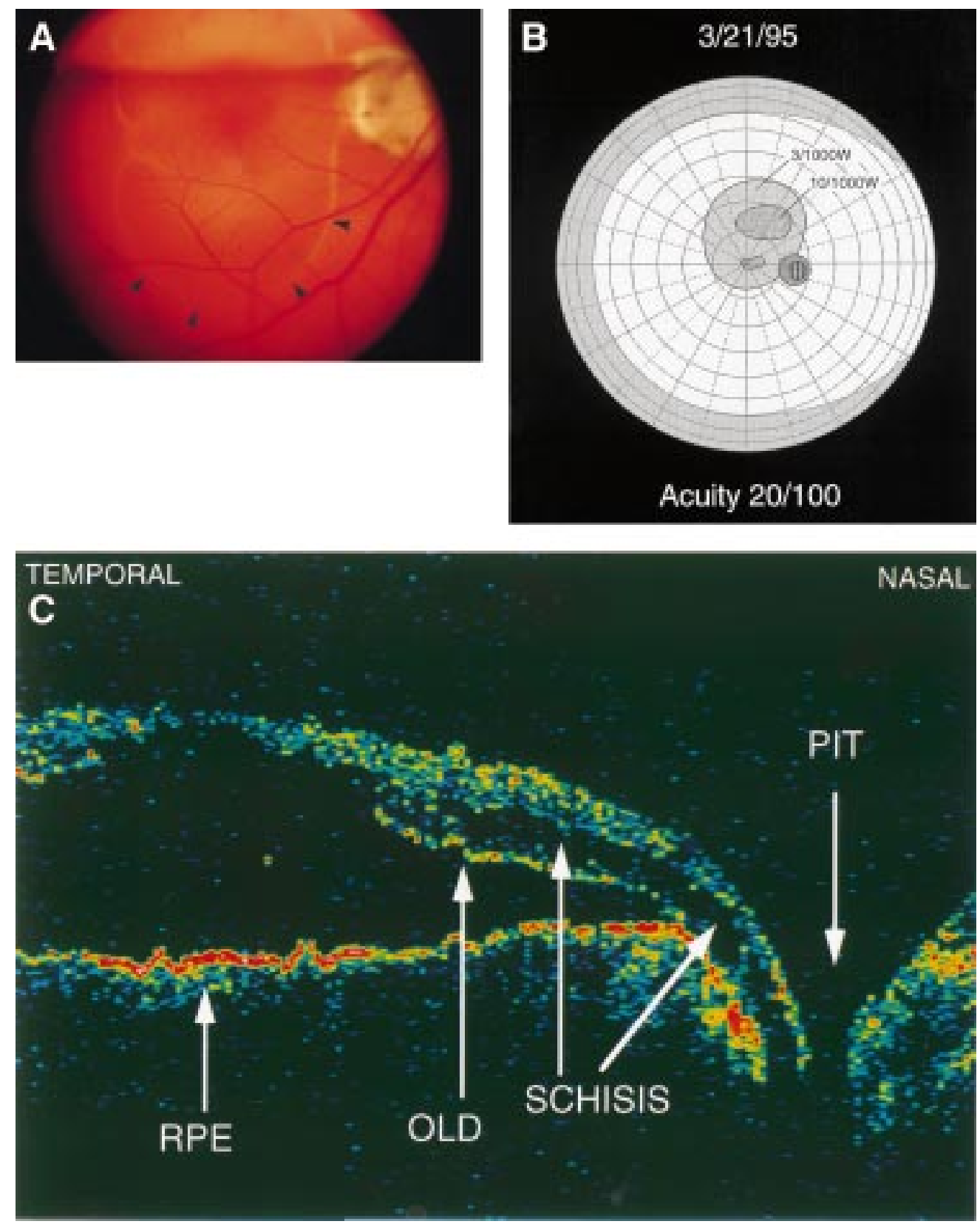

Figure 2 (A) Posterior pole (patient no 1) at 1 week after the injection of $C_{2} F_{6}$ : the gas meniscus is at the upper border of the disc. The OLD has been displaced below the disc (arrowheads). (B) Central field at 1 week: It demonstrates a dense scotoma $(10 / 1000 \mathrm{~W})$ consistent with the OLD, that has been displaced above the posterior pole. A less dense scotoma $(3 / 1000 \mathrm{~W})$ is consistent with the extent of the ILS. (C) OCT of the posterior pole at 1 week. It reveals the retinal elevation diminished by half. The schisis-like cavity connects to the disc pit. The OLD attaches to the retinal pigment epithelium at the edge of the disc. RPE = retinal pigment epithelium; OLD = outer layer detachment; - horizontal section through macula and disc.

OCT at 1 week revealed the central elevations to be diminished by half and demonstrated convincingly that the schisis-like cavity connected to the optic disc pit and that the OLD was attached to pigment epithelium at the edge of the disc. A hole in the OLD at the macula was apparent (Fig 2).

After 3 weeks of gas massage and compression, the retina at the posterior pole appeared flat. A retinal elevation created by displaced subretinal and intraretinal fluid, was apparent below the inferior vascular arcade. The visual field demonstrated a dense scotoma between $15^{\circ}$ and $25^{\circ}$ above fixation that corresponded with the displaced OLD. Surrounding it was a less dense scotoma consistent with the more widespread ILS. The visual field indicated that the ILS persisted across the posterior pole. The visual acuity was 20/40. An OCT directed horizontally through the fovea and the disc pit revealed most of the OLD in contact with pigment epithelium. The ILS maintained a shallow elevation that connected to the optic disc pit (Fig 3).

The improvement in visual acuity persisted for 1 month. In the second month the patient reported deterioration. Examination revealed the visual acuity reduced to 20/70. Biomicroscopy and stereoscopic photographs revealed persistence of the displaced retinal elevation 

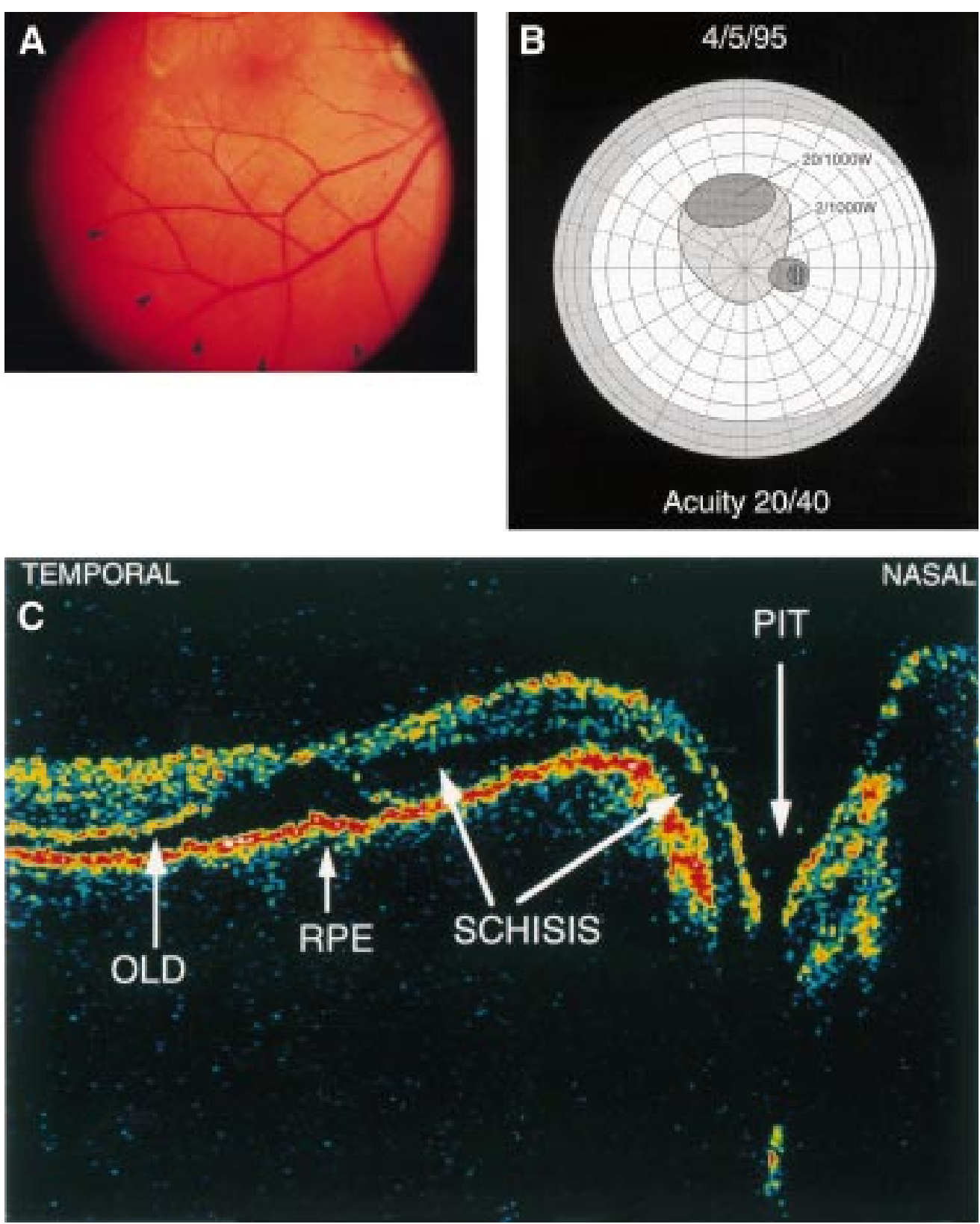

Figure 3 (A) Posterior pole (patient 1) at 3 weeks. The OLD is displaced below the inferior temporal vein (arrowheads). (B) Central visual field at 3 weeks. It demonstrates a dense scotoma (20/1000W) between $15^{\circ}$ and $25^{\circ}$ above fixation consistent with the OLD. A less dense scotoma $(2 / 1000 \mathrm{~W})$ persists in the posterior pole. (C) OCT at 3 weeks. It reveals the retinal layers almost attached to the pigment epithelium. A hole in the OLD at the macula is apparent. The schisis-like cavity persists and is confluent with the optic disc pit. RPE = retinal pigment epithelium; OLD = outer layer detachment; - horizontal section through macula and disc.

below the inferior vascular arcade, but an increase in the elevation of the ILS centrally. An OLD was not apparent, but the visual field demonstrated a dense central scotoma $5^{\circ}$ in diameter. OCT revealed the recurrence of an OLD in the macula. Over the ensuing year the visual acuity decreased to $20 / 100$. The patient has deferred a second procedure.

PATIENT 2

A 22 year old man who was training to be a golf professional noted diminished vision in his left eye and reduced ability to hit a golf ball effectively for 1 month. Examination revealed the visual acuity in his left eye to be $20 / 100$. He was 2 dioptres hyperopic, did not wear glasses, and stated that his visual acuity, until the past month, had been 20/20 in each eye. The retinal examination revealed a temporal optic disc pit. Extending from the disc pit was a relatively transparent ILS about $20^{\circ}$ in diameter. External to it was an OLD centred on the macula that was about $10^{\circ}$ in diameter. The visual field was consistent with the biomicroscopic appearance. OCT demonstrated the two layer structure of the maculopathy.

The affected eye was injected with $0.6 \mathrm{ml}$ of $\mathrm{C}_{2} \mathrm{~F}_{6}$. At 3 days the meniscus of the bubble was at the superior edge of the disc and the patient began rotation exercises and positioning. At 2 weeks the ILS was only a disc diameter below the inferior vascular arcade. The extent of the OLD and the visual acuity were unchanged. 

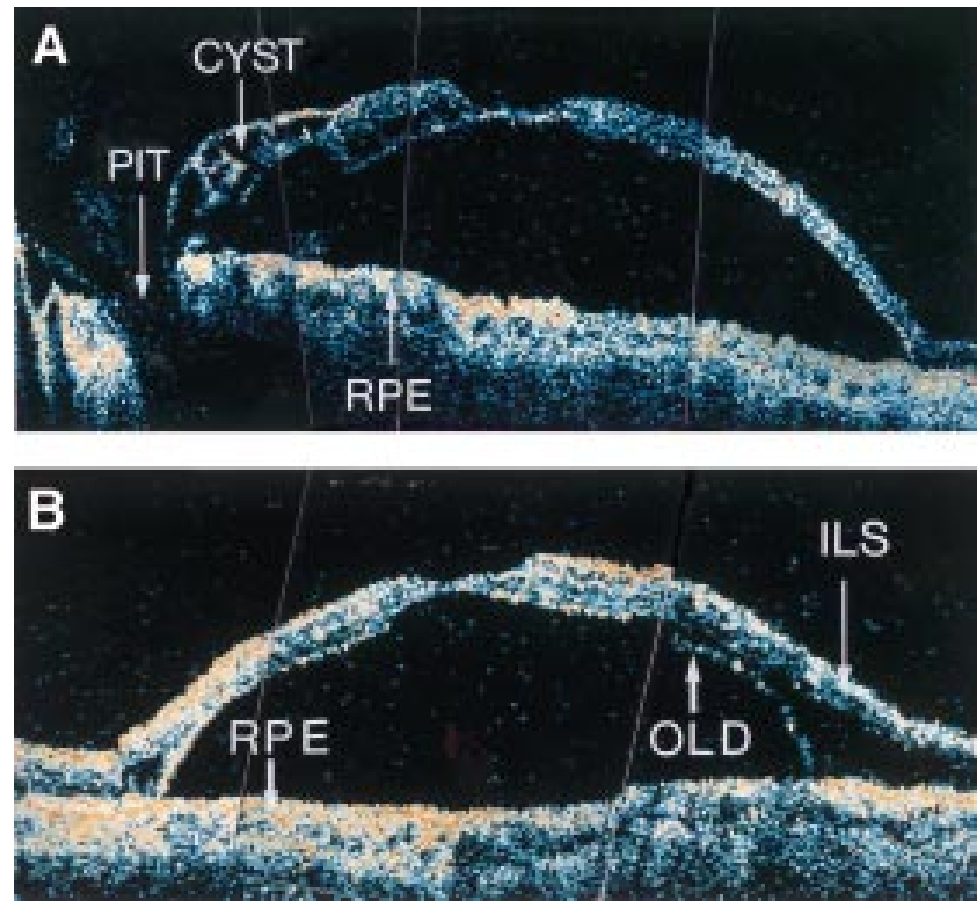

Figure 4 (A) OCT demonstrating recurrence of the OLD in patient no 3 five years after gas displacement. The OLD is in close approximation to the ILS except at its margins. Nasally the ILS is cystic. The visual acuity had diminished from 20/40 to 20/100. RPE = retinal pigment epithelium; - horizontal section through macula and disc. (B) Vertical section through the macula of patient no 3. RPE = retinal pigment epithelium; $O L D=$ outer layer detachment; ILS = inner layer separation.

OCT also confirmed that the elevation of the OLD was unchanged. Two months later a second attempt to displace his macular elevations with gas failed. His visual acuity remained at $20 / 100$ and he has given up his plans to become a golf professional.

PATIENT 3

A 55 year old Armenian clergyman first appeared with classic optic disc pit maculopathy in 1983 . He was the seminal case in the original report on retinoschisis in optic disc pit maculopathy. ${ }^{1} \mathrm{He}$ was treated in that year with laser applications to the edge of the disc, a gas injection, and face down positioning for 1 week, with no effect.

Nine years later in 1992 we asked him to try the gas displacement procedure. His visual acuity was $20 / 100$ in the affected eye. The extent of the ILS was unchanged. The OLD had enlarged to $15^{\circ}$ and there was a hole in the OLD at the macula. The pigment epithelium in the macula was grossly mottled and thought to be dystrophic. The eye was injected with 0.4 $\mathrm{ml}$ of $\mathrm{C}_{2} \mathrm{~F}_{6}$ and the patient instructed about rotations and positioning. Visual acuity improved steadily until by the fourth week he was able to read 20/50. At 6 months he read 20/40. The OLD at the posterior pole appeared flat and the macular hole was not perceptible. An extensive ILS and OLD were apparent below the inferior arcade. The patient was seen at 6 month intervals. The visual acuity diminished to $20 / 50$ in the third year and to $20 / 100$ in the fifth year. OCT, which was then available, revealed a broad separation of the OLD in the macula that appeared in apposition to the ILS except at its margins (Fig 4). The displaced
ILS and OLD were still evident below the inferior vascular arcade, but were less elevated.

\section{Results}

OCT of three patients with optic disc pit maculopathy has provided additional evidence that the apparent serous detachment of the macula, that connects to the pit, is a separation of the inner layers of the retina. There is an OLD that centres on the macula and does not connect to the disc pit. $^{8-10}$ OCT has also provided evidence that an improvement in central vision coincides with a flattening of the OLD in the macula.

The visual acuity of the first patient improved from $20 / 100$ to $20 / 40$ and the improvement coincided with OCT evidence of reattachment of the OLD. Visual acuity diminished to $20 / 70$ a month later and OCT confirmed the reappearance of a shallow OLD centrally. The second patient showed no improvement after two 3 week periods of attempted gas displacement. OCT revealed that the OLD and to a lesser extent the ILS resisted gas displacement. The third patient with a 9 year presence of optic disc pit maculopathy, which had been treated initially with laser coagulation to the edge of the disc with no effect, improved from $20 / 100$ to $20 / 40$ after gas displacement. He maintained improved visual acuity until the fifth year. An OCT in the fifth year demonstrated recurrence of the OLD.

\section{Discussion}

OCT has revealed that the retinal elevation associated with optic disc pits resists gas compression and massage, unlike pneumatic displacement of rhegmatogenous detachment. This is probably because of the schisis-like nature of the ILS. At 1 week, OCT of patient no 1 revealed the ILS still separated by 800 to $1000 \mu \mathrm{m}$. This may explain the frequent failure of photocoagulation to the edge of the disc, in combination with gas compression, to create an adhesive barrier for fluid from the pit. Laser applications are unlikely to close the space maintained by the ILS at 1 week as depicted in Figure 2. We speculate that the ILS which persists as a shallow elevation even after 3 weeks of massage and compression is providing a conduit for fluid from the disc pit to the displaced reservoir below the inferior vascular arcade.

The OCT images should eliminate one of the theories about the origin of the subretinal fluid in the optic disc pit syndrome. The persistence of the retinal elevation at 1 week, in the presence of an intraocular gas bubble that has tamponaded the elevation and the optic disc pit, make it unlikely that retinal breaks at the edge of the disc are transferring fluid from the vitreous to beneath the retina. ${ }^{11-13}$

Gas displacement of the OLD in optic disc pit maculopathy has provided an improvement in visual acuity in nine of the 12 patients treated in this manner. The improvement, however, is temporary. OCT of the last three patients in our series has confirmed that the diminished acuity coincides with the reappearance of an OLD in 
the macula. We think this may be because the reservoir created by the gas displacement flattens and closes with time while the flow from the disc pit remains constant. A more permanent reservoir for flow from the optic disc pit might be the orbit with an egress into it, provided by an optic nerve fenestration.

Presented in part at the 94th Session of the German Ophthalmological Society, 22 September 1996 in Mannheim, Germany.

The authors want to thank Dr Robert Ritsch for providing the OCT facilities of his laboratory.

1 Lincoff H, Lopez R, Kreissig I, et al. Retinoschisis associated with optic nerve pits. Arch Ophthalmol 1988;109:61-7.

2 Lincoff $\mathrm{H}$, Yannuzzi L, Singerman L, et al. Improvement in visual function after displacement of the retinal elevations emanating from optic pits. Arch Ophthalmol 1993;111: 1071-9.

3 Huang D, Swanson EA, Lin CP, et al. Optical coherence tomography. Science 1991;254:1178-81.
4 Puliafito CA, Hee MR, Lin CP, et al. Imaging of macular diseases with optical coherence tomography. Ophthalmology 995:102:217-29.

5 Kreissig I. The balloon-gas-procedure. Another move towards minimum surgery. Dev Ophthalmol 1987;13:99106.

6 Kreissig I, Kamel H. The balloon-gas procedure: a technique for repair of retinal detachments requiring large volumes of gas. Eur f Ophthalmol 1991;1:11-16.

7 Lincoff A, Haft D, Liggett P, et al. Intravitreal expansion of perfluorocarbon bubbles. Arch Ophthalmol 1980;98:1646-9.

8 Krivoy D, Gentile R, Liebmann JM, et al. Imaging congenital optic disc pits and associated maculopathy using optic coherence tomography. Arch Ophthalmol 1996;114:165-70.

9 Lincoff H, Schiff W, Krivoy D, et al. Optic coherence tomography of optic disc pit maculopathy. Am f Ophthalmol 1996;122:264-6.

10 Rutledge BK, Puliafito CA, Duker JS, et al. Optical coherence tomography of macular lesions with optic nerve

11 Brown GC, Shields JA, Patt BE, et al. Congenital pit of the optic nerve head. I Experimental studies in collie eyes. Arch optic nerve head. I Experime

12 Sugar HS. Congenital pits in the optic disc. Am f Ophthalmol 1967;63:298-307.

13 Brockhurst RJ. Optic pit and posterior retinal detachment. Trans Am Ophthalmol Soc 1975;73:264-91. 